\title{
STUDY OF THE TUBERCLE OF ZUCKERKANDL AND ITS RELATION TO RECURRENT LARYNGEAL NERVE
}

Vinaya Chandra P. $H^{1}$

${ }^{1}$ Professor, Department of Anatomy, Kannur Medical College, Anjarakandy, Kannur, Kerala.

\section{ABSTRACT}

\section{BACKGROUND}

Objectives- Tubercle of Zuckerkandl (TZ) is the posterolateral extension of thyroid lobe. TZ is the important surgical landmark for identification of recurrent laryngeal nerve (RLN). The objective of the study was to locate the TZ, report the incidence and type of $\mathrm{TZ}$ and to evaluate it as a guide to localise RLN.

\section{MATERIALS AND METHODS}

In a descriptive cadaveric study, 14 specimens with 28 sides were studied (12 males and 2 females). After exposing thyroid gland, neurovascular structures at inferior pole were noted. Lobes were medially reflected and location, extent and type of Tubercle of Zuckerkandl (TZ) was noted. TZ was classified into four categories. Type 1 if unrecognisable, type 2 if only a thickening of lateral edge of the thyroid lobe present, type 3 if a small projection is seen less than $1 \mathrm{~cm}$ and type 4 if projection greater than $1 \mathrm{~cm}$ is appreciated.

\section{RESULTS}

Of the 28 hemi-sections of head and neck examined, TZ was appreciated in 21 specimens. Type 2 in four (14.2\%), type 3 in six (25) and type 4 in eleven (39.2\%) of the cases was noted. Among 17 specimens with appreciable TZ (type 3 and type 4 combined), RLN was present posterior to TZ in 13 specimens. In rest five specimens, RLN was medial to TZ.

\section{CONCLUSION}

Posterolateral extension of lateral lobes of thyroid gland or TZ is found in majority of the cases. In specimens with appreciable TZ (type 3 and type 4 combined), RLN was present posterior to TZ.

\section{KEYWORDS}

Thyroid Gland Anatomy; Inferior Thyroid Artery; Recurrent Laryngeal Nerve; Thyroidectomy; Tubercle of Zuckerkandl.

HOW TO CITE THIS ARTICLE: Chandra VPH. Study of the tubercle of Zuckerkandl and its relation to recurrent laryngeal nerve. J. Evolution Med. Dent. Sci. 2017;6(32):2639-2641, DOI: 10.14260/Jemds/2017/569

\section{BACKGROUND}

Tubercle of Zuckerkandl (TZ) is the posterolateral extension of thyroid lobe. Condensation of thyroid parenchyma at the level of cricothyroid junction on the posterolateral aspect of the thyroid gland forms a tubercular projection and is referred as tubercle of Zuckerkandl. ${ }^{1}$ It was first described by Otto Madelung and later by Emil Zuckerkandl. Presence of TZ was thought to be an embryological event. Thyroid gland develops as an endodermal ingrowth of cells from foramen caecum. Such growth later forms thyroglossal duct. Right and left lobes of the gland develop as outgrowths from this duct. In addition, there is a lateral thyroid component arising from $4^{\text {th }}$ pharyngeal cleft and ultimobranchial body. This fuses with the lobes and forms TZ. Microscopically TZ has maximum C cells in addition to thyroid follicles. ${ }^{2,3}$ Superior parathyroid gland is located posterior to the TZ. Superior parathyroid gland is of relatively more constant position than the inferior parathyroid gland.

Financial or Other, Competing Interest: None.

Submission 15-03-2017, Peer Review 08-04-2017,

Acceptance 14-04-2017, Published 20-04-2017.

Corresponding Author:

Dr. Vinaya Chandra P. H,

Professor, Department of Anatomy,

Kannur Medical College,

Anjarakandy,

Kannur-670612.

E-mail: vinayaherle@gmail.com

DOI: $10.14260 /$ jemds $/ 2017 / 569$
Surgical importance of TZ lies in the fact that it can be a landmark for identification of Recurrent laryngeal nerve (RLN). ${ }^{4-8}$ Nerve enters larynx behind TZ. ${ }^{9}$ Visual identification and demonstration of RLN is compulsory in all thyroidectomy surgeries. Such identification minimises the injury and preserves the nerve function. For identification of RLN, knowledge about its anatomical position, landmarks of identifications and variations is a must.10 The branches of inferior thyroid artery pass superficially anterolateral to RLN. The TZ and superior parathyroid glands lie within a superficial fascial plane. RLN is located in a deeper plane within ligament of Berry. ${ }^{9}$ RLN injuries are more common within $2 \mathrm{~cm}$ of extra-laryngeal course where it is related to inferior thyroid artery trunk. ${ }^{11}$ Unilateral injury to RLN causes hoarseness and cough. Bilateral injury to RLN results in dyspnoea and aphonia. ${ }^{12}$ RLN injury is one of the important medical dispute cases associated with thyroid related interventions. ${ }^{13}$ Despite a clear protocol of nerve identification and preservation, the iatrogenic injury to nerve ranges from $0.5-5 \%$ of the thyroidectomy cases. This is accounted by higher anatomical variations including unusual extra-laryngeal branches, intertwining of the branches of RLN and inferior thyroid artery, non-recurrent laryngeal nerve. ${ }^{14}$ Intraoperative neuro-monitoring for early localisation and identification of recurrent laryngeal nerve during thyroid surgery is advocated.15,16

As the identification of RLN is of prime significance, many anatomists and surgeons consider importance during thyroidectomy, anatomical landmarks for its localisation in 
detail.1,4-6 The objective of the study was to locate the TZ, report the incidence and type of $\mathrm{TZ}$ and to evaluate it as a guide to localise RLN. There are few cadaveric studies evaluating TZ and RLN relationship.

\section{MATERIALS AND METHODS}

In a descriptive cadaveric study, 28 head and neck specimens of 14 cadavers were studied (12 males and 2 females). All cadavers were unclaimed bodies from district hospital and age range could not be ascertained. All cadavers were formalin fixed via femoral artery and neck was not dissected for the purpose of embalming.

Anterior triangle was dissected via a midline incision. After reflecting platysma and the related fascia, strap muscles were cut in the middle and reflected. Thyroid gland inferior pole is exposed and neurovascular structures were studied. After reflecting lobes medially, location, extent and type of Tubercle of Zuckerkandl (TZ) was noted and documented. TZ was classified into four categories. Type 1 if unrecognisable, type 2 if only a thickening of lateral edge of the thyroid lobe present, type 3 if a small projection is seen less than $1 \mathrm{~cm}$ and type 4 if projection greater than $1 \mathrm{~cm}$ is appreciated. This is a modified classification of scheme proposed by Pelizzo et al. ${ }^{7}$ Location and course of RLN was noted.

\section{RESULTS}

Of the 28 hemisections of head and neck examined, TZ was appreciated in 21 specimens. No projection from the lateral wall was noted in six sides. A small projection amounting to slight deviation of lateral border was noted in four specimens. Type 3 variety with less than a cm projection was noted in six sides. Type $4 \mathrm{TZ}$ with more than a $\mathrm{cm}$ was noted in eleven sides (table 1 and figure 1). In all specimens where $\mathrm{TZ}$ was present, it was unilobar.

\begin{tabular}{|c|c|}
\hline Tubercle of Zuckerkandl (TZ) & Noted in \\
\hline Type 1 (Not appreciated) & $6(21.4 \%)$ \\
\hline Type 2 (Small projection) & $4(14.2 \%)$ \\
\hline Type $3(<1 \mathrm{~cm})$ & $6(25)$ \\
\hline Type $4(>1 \mathrm{~cm})$ & $11(39.2 \%)$ \\
\hline $\begin{array}{c}\text { Table 1. Tabulation of Tubercle of Zuckerkandl types } \\
\text { noted During the Study. (n = 28) }\end{array}$ \\
\hline
\end{tabular}

In all specimens, RLN was identified in the tracheoesophageal groove very close to inferior thyroid artery near the thyroid lobe. Among 17 specimens with appreciable TZ (type 3 and type 4 combined), RLN was present posterior to $\mathrm{TZ}$ in 13 specimens. In rest five specimens, RLN was medial to TZ. There were no branches of RLN above TZ.

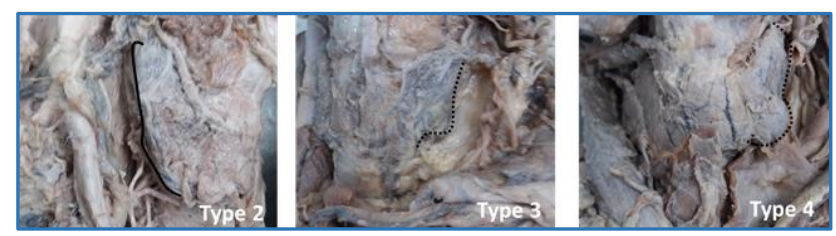

Figure 1. Tubercle of Zuckerkandl types, Type 2: Small Projection, Type 3: $<1 \mathrm{~cm}$ Projection, Type 4: >1 cm Projection

\section{DISCUSSION}

Surgeons develop their own way to identify the RLN during thyroid surgeries. Knowledge of anatomical landmarks of tracheoesophageal groove, inferior thyroid artery, ligament of Berry, tubercle of Zuckerkandl and inferior constrictor muscle are the basis of methods of RLN identification. ${ }^{1}$ Presence of TZ reported in the earlier studies varies greatly. Popescu $^{8}$ reported 73\%, Irawati ${ }^{4}$ reported 90\%, Mehmood ${ }^{5}$ et al reported $62 \%$, and Rajapaksha ${ }^{17}$ et al noted in $90 \%$ of the patients. Only Chintamani ${ }^{18}$ et al (35\%) and James ${ }^{6}$ et al (43\%) reported occurrence of TZ in less than half the of the patients observed. Many of the studies were done in patients undergoing thyroid surgeries for various reasons. There is a possibility that observations of occurrence of $\mathrm{TZ}$ is skewed as the patients with thyroid disorder have altered thyroid anatomy. Rajapaksha et al have measured the closest distance between TZ and RLN and found that nerve is located within 1 cm from TZ.17

With regard to grade of TZ reported in present study, we concur with Irawati,4 ${ }^{4}$ Mehmood $^{5}$ and James. ${ }^{6}$ In all these studies, more than half of TZ is found to of grade 3 and grade 4. However, Rajapaksha ${ }^{17}$ and Chintamani ${ }^{18}$ reported smaller TZ (grade 2) in more than half of the patients they have observed. In all the studies, including ours, RLN was found medial to TZ. However, in a report by Gurleyik E et al, five cases were reported where RLN was found anterior to TZ. ${ }^{19}$ Many surgeons opine that grading of $\mathrm{TZ}$ is not of much practical significance as the surgeries are done on the enlarged thyroid glands. ${ }^{11,18}$ It is advocated that $\mathrm{TZ}$ is carefully dissected and separated while performing thyroidectomy. While working on enlarged thyroid gland, incomplete dissection of TZ may result in recurrence of the disease. $3,4,8$

Variations of TZ is reported by Sadacharan et al. They noted a giant tubercle of Zuckerkandl with retrosternal extension encountered during total thyroidectomy. ${ }^{20}$ No such retrosternal extensions were noted in the present study.

\section{CONCLUSION}

Posterolateral extension of lateral lobes of thyroid gland or $\mathrm{TZ}$ is found in majority of the cases. It can be used as a useful anatomical landmark to locate RLN.

\section{REFERENCES}

[1] Bhargav PR. Salient anatomical landmarks of thyroid and their practical significance in thyroid surgery: a pictorial review of thyroid surgical anatomy (revisited). Indian J Surg 2014;76(3):207-11.

[2] Ritchie JE, Balasubramanian SP. Anatomy of the pituitary, thyroid, parathyroid and adrenal glands. Surgery 2014;32(10):499-503.

[3] Nussey S, Whitehead S. The thyroid gland. Chapter 3. In: Endocrinology an integrated approach. Oxford: BIOS Scientific Publishers 2001. https://www.ncbi.nlm.nih.gov/books/NBK28/

[4] Irawati N, Vaish R, Chaukar D, et al. The tubercle of zuckerkandl : an important landmark revisited. Indian J Surg Oncol 2016;7(3):312-5.

[5] Mehmood Z, Khan U, Bokhari I, et al. Zuckerkandl tubercle: an important landmark in thyroid surgery. J Coll Physicians Surg Pak 2015;25(7):495-7. 
[6] James YE, Doleagbenou A, Kassegne I, et al. Zuckerkandl's tubercle: incidence and relationship with the inferior laryngeal nerve. Morphologie 2014;98(323):171-5.

[7] Pelizzo MR, Toniato A, Gemo G. Zuckerkandl's tuberculum: an arrow pointing to the recurrent laryngeal nerve (constant anatomical landmark). J Am Coll Surg 1998;187(3):333-6.

[8] Popescu R, Ponoran D, Ignat O, et al. Monitoring the laryngeal nerves during thyroidectomy. Initial 115 cases experience. Chirurgia (Bucur) 2015;110(4):32732.

[9] Serpell JW. New operative surgical concept of two fascial layers enveloping the recurrent laryngeal nerve. Ann Surg Oncol 2010;17(6):1628-36.

[10] Zakaria HM, Al Awad NA, Al Kreedes AS, et al. Recurrent laryngeal nerve injury in thyroid surgery. Oman Med J 2011;26(1):34-8.

[11] Jiang Y, Gao B, Zhang X, et al. Prevention and treatment of recurrent laryngeal nerve injury in thyroid surgery. Int J Clin Exp Med 2014;7(1):101-7.

[12] Misron K, Balasubramanian A, Mohamad I, et al. Bilateral vocal cord palsy post thyroidectomy: lessons learnt. BMJ Case Reports 2014. DOI: 10.1136/bcr2013-201033.

[13] Marcus B, Edwards B, Yoo S, et al. Recurrent laryngeal nerve monitoring in thyroid and parathyroid surgery: the University of Michigan experience. Laryngoscope 2003;113(2):356-61.
[14] Chiang FY, Lu IC, Chen HC, et al. Anatomical variations of recurrent laryngeal nerve during thyroid surgery: how to identify and handle the variations with intraoperative neuromonitoring. Kaohsiung J Med Sci 2010;26(11):575-83.

[15] Chiang FY, Lu IC, Chen HC, et al. Intraoperative neuromonitoring for early localization and identification of recurrent laryngeal nerve during thyroid surgery. Kaohsiung J Med Sci 2010;26(12):633-9.

[16] Dralle H, Sekulla C, Lorenz K, et al. Intraoperative monitoring of the recurrent laryngeal nerve in thyroid surgery. World J Surg 2008;32(7):1358-66.

[17] Rajapaksha A, Fernando R, Ranasinghe N, et al. Morphology of the tubercle of zuckerkandl and its importance in thyroid surgery. Ceylon Med J 2015;60(1):23-4.

[18] Chintamani. Friend or foe of a thyroid surgeon?-the tubercle of Zuckerkandl. Indian J Surg 2013;75(5):337-8.

[19] Gurleyik E, Gurleyik G. Incidence and surgical importance of Zuckerkandl's tubercle of the thyroid and its relations with recurrent laryngeal nerve. ISRN Surg 2012;2012:1-5.

[20] Sadacharan D, Mahadevan S, Muthukumar S, et al. A rare giant tubercle of zuckerkandl with retrosternal extension encountered during total thyroidectomy. BMJ Case Reports 2015. DOI: 10.1136/bcr-2015210307. 